\title{
Low frequency acoustic energy harvesting adopting slit Helmholtz resonator
}

\author{
Ming Yuan ${ }^{1}$, Xiaohui Wang ${ }^{2}$, Zhenjun Ding ${ }^{3}$ \\ ${ }^{1}$ School of Automation, Nanjing University of Posts and Telecommunications, Nanjing, P. R. China \\ ${ }^{2}$ Anhui Wanzte P\&T Co., Ltd, Nanjing, P. R. China \\ ${ }^{3}$ Beijing Institute of Structure and Environment Engineering, Beijing, P. R. China \\ ${ }^{1}$ Corresponding author

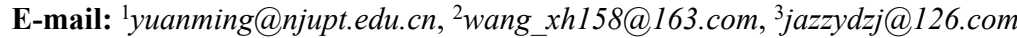

Received 19 September 2018; accepted 26 September 2018

DOI https://doi.org/10.21595/vp.2018.20245

Check for updates

Copyright $(2018$ Ming Yuan, et al. This is an open access article distributed under the Creative Commons Attribution License, which permits unrestricted use, distribution, and reproduction in any medium, provided the original work is properly cited.

\begin{abstract}
In this study, a novel acoustic energy harvesting (AEH) device is proposed to realize low frequency acoustic energy harvesting in broadband. Based on the classical Helmholtz resonator, a novel AEH device with slits is proposed. The AEH components and mechanisms are analyzed, followed by an experimental evaluation of the fabricated sample. Experimental results show that for the proposed AEH device, the bandwidth near the acoustic resonant frequency is extended, which can be attributed to the thermos-viscous damping mechanism. This type of attribute is favorable for resonance tuning and increasing the adaptability of the AEH device. Under $100 \mathrm{~dB}$ sound pressure level (SPL) excitation, the measured harvested electrical power is $2.4 \mu \mathrm{W}$.
\end{abstract}

Keywords: acoustic energy harvesting, piezoelectric, low frequency, Helmholtz resonator.

\section{Introduction}

Acoustic energy, which belongs to one kind of environmental energies, is abundant around noise sources, such as power plants, aircrafts, fluid ducts, vehicles, railways, and factories. Large decibels of noise bring harmful effects to human beings and are usually unwanted. Therefore, many methods have been developed to suppress noise through insulation or dissipation mechanisms. On the other hand, sound energy can be harvested and transformed into electrical energy for low power electrical application.

Among the frequency range, low frequency acoustic energy draws the most attention. Firstly, the major environmental noise sources generate low frequency noise rather than high frequency noise, making it abundant. Secondly, the wavelength of low frequency noise in air can be meters long, causing such type of noise to have a long propagation distance and having fewer restrictions that need to be considered for the acoustic energy harvester design.

In general, the density of acoustic energy is low; an acoustic resonator is necessary to increase the incident sound pressure. This strategy can make the energy conversion part become sufficiently excited, which is favorable to improve the acoustical-electrical energy conversion efficiency. Therefore, many approaches have been developed, such as Helmholtz resonator based $\mathrm{AEH}$ [1-3], quarter wavelength resonator based AEH [4, 5], and acoustic metamaterial based AEH $[6,7]$.

Although fruitful research findings have been generated in recent years, there are still some gaps to fill. For instance, the acoustic resonance usually occurs in a narrow band, and the harvested power could be substantially decreased when the excitation frequency is off the acoustic resonant frequency. Previous studies also show that if the mechanical resonance is tuned to be the same as the acoustical resonant frequency, the energy harvesting efficiency can be further improved. However, the tuning process can be very complex.

Making the AEH device has a larger resonant band can be a feasible solution to improve the device's adaptability. To solve this problem, a novel AEH device is proposed in this study. The components of the device are described in Section 2 and the performance of the proposed device 
is evaluated in Section 3 via experimental study. The obtained conclusions are summarized and presented in Section 4.

\section{Proposed AEH design}

As shown in Fig. 1, the proposed AEH device belongs to a modified version of classical Helmholtz resonator.

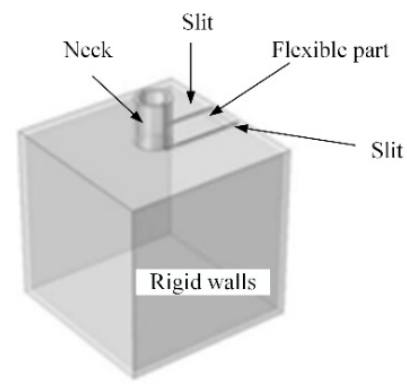

Fig. 1. Schematic diagram of the proposed AEH device

As shown in Fig. 1, the proposed AEH device belongs to a modified version of the classical Helmholtz resonator. Compared with the classical configuration, the modifications are given as follows: (1) At the upper surface, a flexible metallic cantilever is adopted to replace a portion of the original rigid surface. (2) A PZT-5H patch is bonded near the fixed end of the cantilever beam. (3) At the beam's free terminal, one half of the resonator's neck is adhered to the beam, the other part of the neck is adhered on the rigid top surface.

During the prototype fabrication, the dimensional parameter of the metallic beam is $50 \times 30 \times 0.6 \mathrm{~mm}$, and the material type is copper. The dimensional parameter of PZT- $5 \mathrm{H}$ patch is $30 \times 20 \times 0.2 \mathrm{~mm}$. The dimensional parameter of the cavity is $80 \times 80 \times 90 \mathrm{~mm}$, and the material type is stainless steel. The length of the neck is $20 \mathrm{~mm}$, with an inner diameter of $8 \mathrm{~mm}$ and thickness of $5 \mathrm{~mm}$, which is fabricated via 3D printing technique.

The photograph for the prototype is shown in Fig. 2.

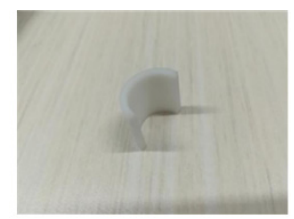

a) Plastic neck component

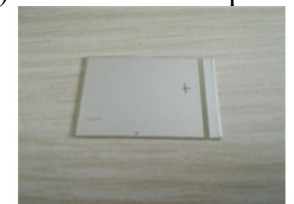

b) Copper component

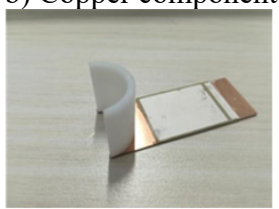

d) Assembled cantilever

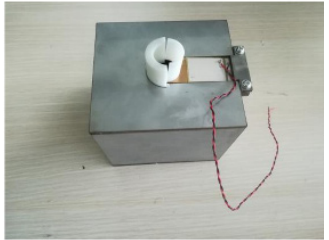

e) Assembled prototype

c) Piezoelectric path component

Fig. 2. Prototype of the proposed AEH harvester

Several issues are considered to design this type of AEH harvester.

Firstly, compared with the previous AEH designs, a cantilever beam configuration is anticipated to generate a large stain, which is favorable to enhance the mechanical-electrical energy conversion efficiency.

Secondly, the half part of the neck, which is bonded on the cantilever beam, can be served as proof mass, which helps to increase the energy harvesting performance in future steps, and the 
mechanical resonance frequency can be tuned via alternating the structural parameters of the neck.

Finally, the assembled AEH structure is similar to the classical Helmholtz resonator, and acoustic resonance is anticipated at a specific frequency. However, due to the narrow slits existing on the device, the introduced viscous losses can be substantial. The introduced acoustic damping helps to generate a broadband effect around the resonant frequency.

The thickness of viscous boundary layer can be calculated as:

$\delta=\sqrt{\frac{\mu}{\pi f \rho_{0}}}$,

where $\mu$ is the dynamic viscosity, $f$ is frequency, and $\rho_{0}$ is the air density.

For instance, at $100 \mathrm{~Hz}$, the calculated value in the low frequency range is $0.22 \mathrm{~mm}$. This large value indicates that the contribution of the viscous loss is significant in the low frequency range.

\section{Experimental setup and measured results}

Band limited white noise is used for the excitation signal. The fabricated sample is placed in front of a 6.5-inch loudspeaker (HiVi SR 6.5) and a microphone sensor (G.R.A.S $40 \mathrm{pp}$ ) is placed into the cavity for sound pressure monitoring.

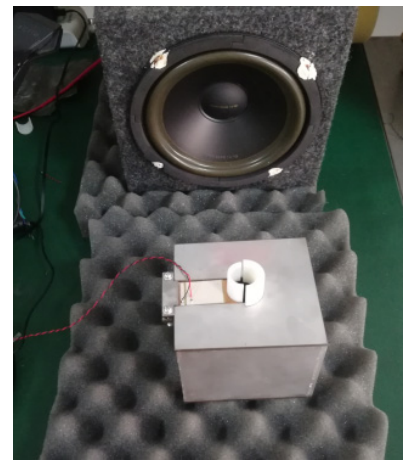

Fig. 3. Experimental test of the proposed AEH structure

For a comparison test, a classical Helmholtz resonator with the same outer dimension is also fabricated, and the two obtained sound pressure level (SPL) spectra are plotted in Fig. 4.

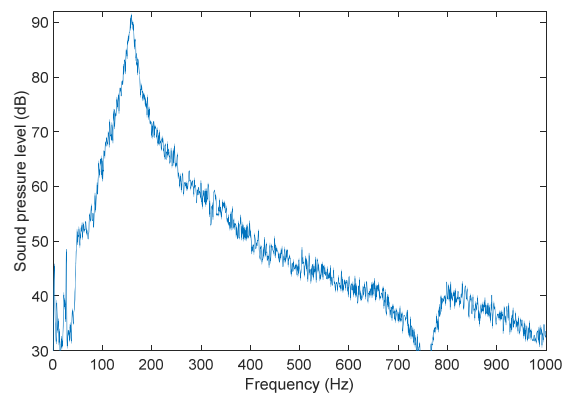

a) Classical Helmholtz resonator

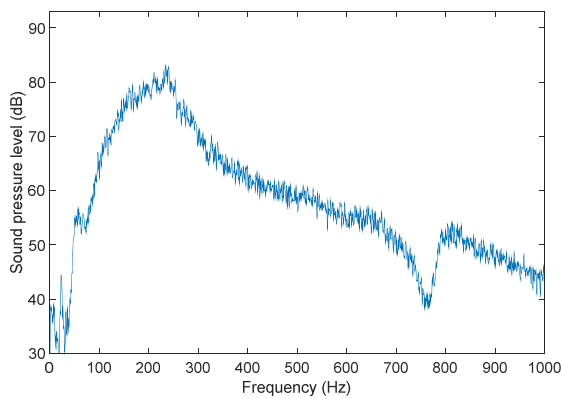

b) Proposed device

Fig. 4. Measured SPL spectra

For the classical Helmholtz resonator, the acoustic resonance occurs at $160 \mathrm{~Hz}$, and the measured SPL peak value is $91 \mathrm{~dB}$. If acoustic cavity is removed, the measured value for that frequency is only $70 \mathrm{~dB}$. Therefore, the sound pressure amplification gain is eleven. In 
comparison, for the proposed AEH structure, the spectrum around the resonance is much flatter, and large sound pressure amplification can be observed at $150 \mathrm{~Hz}$ to $250 \mathrm{~Hz}$. Meanwhile, the identified maximum SPL occurs at $234 \mathrm{~Hz}$. At this frequency, the calculated sound pressure amplification gain is seven.

In further steps, the open loop voltage - which is generated by the piezoelectric patch - is measured. Two cases are considered: (1) only the cantilever beam is excited and (2) the cantilever beam is fixed on the top surface of the cavity and excited. The acoustic excitation strength is kept as constant and the corresponding results are provided in Fig. 5.

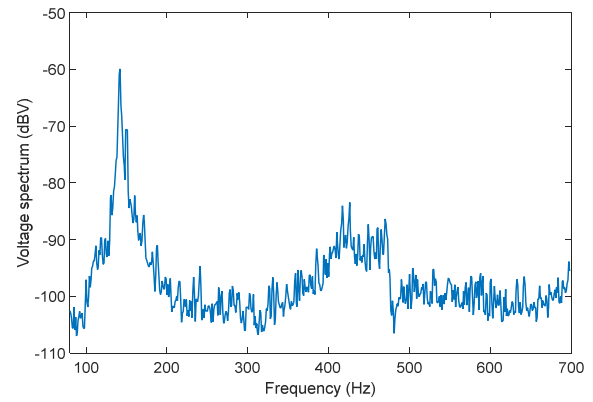

a) Cantilever beam alone

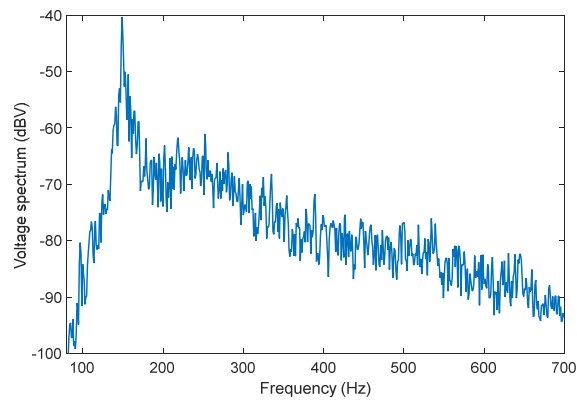

b) Cantilever beam is placed onto the cavity

Fig. 5. Measured voltage spectra

As shown in Fig.5, the measure voltage spectra demonstrate that when the acoustic cavity is added, the voltage is increased by $20 \mathrm{~dB}$. This is attributable to the AEH cavity having large sound pressure amplification bandwidth. Further, the mechanical part can be strongly excited when the resonant frequency of the mechanical part is located on this band. The resonance matching is favorable for the AEH performance boosting.

To evaluate the harvested power, a decade box is wired to the piezoelectric patch. The resistance is varied and realizes resistance matching for maximum power extraction. The voltage on the load is recorded and the harvest power can be calculated as:

$P=\frac{V_{r m s}^{2}}{R}=\frac{\left(\frac{V_{p p}}{2} / \sqrt{2}\right)^{2}}{R}=\frac{V_{p p}^{2}}{8 R^{\prime}}$,

where $V_{r m s}$ is the root mean squared voltage, $V_{p p}$ is the measured peak-peak voltage and $R$ is the corresponding resistance value.

The measured result is given in Fig. 6. It is shown that when the resistance load is equal to $22 \mathrm{k} \Omega$, the maximum harvested power under $100 \mathrm{~dB}$ excitation is $2.4 \mu \mathrm{W}$.

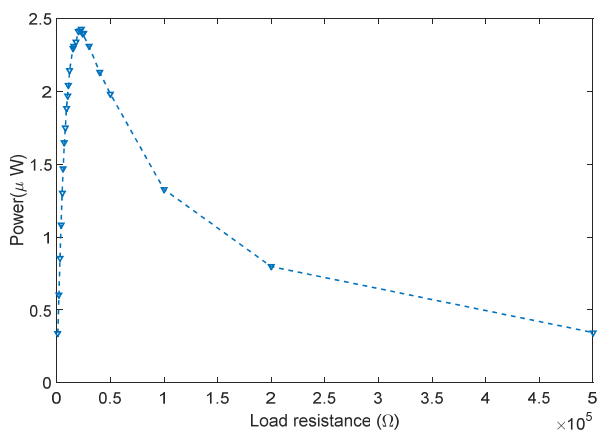

Fig. 6. Harvested power versus different load resistances 


\section{Conclusions}

In this study, a novel AEH structure for the low frequency range is proposed. Compared with the traditional Helmholtz resonator, the added slits assist in enlarging the sound amplification band, which facilitates matching the mechanical and acoustical resonant frequencies. The mechanism is attributed to the viscous loss effect caused by the narrow slits. The harvested power value is limited because the size of mechanical part is small. Besides, the structural parameters can be further optimized to increase the harvested power. These works will be carried out in our study in the near future.

\section{Acknowledgements}

This research is sponsored by National Natural Science Foundation of China (Grant No. 61701250) and the Natural Science Foundation of Jiangsu Province (Grant No. BK20160895).

\section{References}

[1] Yuan M., Cao Z., Luo J., Zhang J., Chang C. An efficient low-frequency acoustic energy harvester. Sensors and Actuators: A. Physical, Vol. 264, 2017, p. 84-89.

[2] Peng X., Wen Y., Li P., Yang A., Bai X. Enhanced acoustoelectric coupling in acoustic energy harvester using dual Helmholtz resonators. IEEE Transactions on Ultrasonics, Ferroelectrics, and Frequency Control, Vol. 60, 2013, p. 2121-2128.

[3] Khan F. U., Izhar Hybrid acoustic energy harvesting using combined electromagnetic and piezoelectric conversion. Review of Scientific Instruments, Vol. 87, Issue 2, 2016, p. 025003.

[4] Li B., Laviage A. J., You J. H., Kim Y. J. Harvesting low-frequency acoustic energy using multiple PVDF beam arrays in quarter-wavelength acoustic resonator. Applied Acoustics, Vol. 74, 2013, p. 1271-1278.

[5] Li B., You J. H., Kim Y.-J. Low frequency acoustic energy harvesting using PZT piezoelectric plates in a straight tube resonator. Smart Materials and Structures, Vol. 22, 2013, p. 055013.

[6] Yuan M., Cao Z., Luo J., Ohayon R. Acoustic metastructure for effective low frequency acoustic energy harvesting. Journal of Low Frequency Noise, Vibration and Active Control, 2018, https://doi.org/10.1177/1461348418794832.

[7] Yuan M., Cao Z., Luo J., Pang Z. Helix structure for low frequency acoustic energy harvesting. Review of Scientific Instruments, Vol. 89, 2018, p. 055002. 